\title{
Preparation, isolation and
} characterization of stage-specific
spermatogenic cells for cellular and
molecular analysis

\author{
Noora Kotaja $^{1,3}$, Sarah Kimmins ${ }^{1,3}$, Stefano Brancorsini ${ }^{1}$, Didier Hentsch ${ }^{1}$, \\ Jean-Luc Vonesch ${ }^{1,}$ Irwin Davidson ${ }^{1}$, Martti Parvinen ${ }^{2} \&$ Paolo Sassone-Corsi ${ }^{1}$ \\ ${ }^{1}$ Institut de Génétique et de Biologie Moléculaire et Cellulaire, CNRS-INSERM, Université Louis Pasteur, B.P. 163, 67404 Illkirch, \\ Strasbourg, France. ${ }^{2}$ Department of Anatomy, University of Turku, FIN-20520, Turku, Finland. ${ }^{3}$ These authors contributed equally to \\ this work. Correspondence should be addressed to P.S.-C. (paolosc@igbmc.u-strasbg.fr).
}

Spermatogenesis constitutes a remarkable program of cell differentiation, which involves dramatic changes in cell morphology, biochemistry and gene expression ${ }^{1,2}$. But the study of male germ cells is complicated by the exceptional organization of the seminiferous epithelium and by the lack of established cell lines that are able to recapitulate any of the multiple differentiation steps of the spermatogenesis program in vitro. Cell types with differences in their sedimentation properties or cell surface markers can be isolated from the whole tissue by various methods, but these methods do not allow accurate identification of all the differentiation stages. As a consequence of strict paracrine regulation by Sertoli cells, spermatogenesis proceeds in synchronized waves along the seminiferous tubules, and every given cross-section of the tubule contains only certain cell types in a specific combination (Fig. 1). The light absorption pattern of a seminiferous tubule, as seen under a dissection microscope, correlates with defined stages of the spermatogenic wave, which makes it possible to isolate specific stages on the basis of their transillumination properties ${ }^{3-6}$. The accuracy of the isolation of specific stages can be improved by combining it with phase-contrast microscopy of live cell preparations ${ }^{7-10}$. The staging of the spermatogenic cycle is best characterized in rat and mouse ${ }^{11,12}$. Here, we describe a method designed to identify, isolate and characterize mouse male germ cells at specific steps of differentiation by transillumination-assisted microdissection. The distinctive morphological features of germ cells, as detected by phase-contrast microscopy, are detailed. Although the method described here generates small quantities of cells and therefore does not allow for further isolation of the different cell types comprising each differentiation stage, the applications of this method are powerful and diverse. It enables the monitoring of spermatogenic differentiation events, and in combination with biochemical analyses, it allows the characterization of the molecular mechanisms governing these processes. This method can be used for the identification of the effects of cytotoxic and environmental factors on male reproductive function, as well as for the rapid and comprehensive diagnosis and characterization of sperm cell defects attributable to infertility. In combination with gene targeting models, it enables the characterization of the critical factors involved in the cell cycle, chromatin dynamics, spermatid differentiation, stem cell biology and fertility.

\section{MATERIALS}

REAGENTS

Phosphate-buffered saline, pH 7.2-7.4 (PBS; Sigma Chemicals)

Immersion oil (Leica) 
Isolation and microdissection of staged segments of the seminiferous tubules

\section{PROCEDURE}

1|Sacrifice a sexually mature mouse, remove the testes and place them in a Petri dish containing PBS. Decapsulate the testis and transfer the seminiferous tubules to a new Petri dish containing PBS.

\section{$\triangle$ CRITICAL STEPS}

2|View the tubules on a transilluminating dissection microscope. Using fine forceps, gently pull apart the tubules, being careful not to cause damage by squeezing or shaking the tubules.

\section{$\Rightarrow$ TROUBLESHOOTING}

A predictable light absorption pattern is produced when the seminiferous tubules are observed under a transilluminating dissection microscope (Supplementary Figure 1 online). The greater the level of spermatid chromatin condensation, the greater is the amount of light absorbed, resulting in the differential appearance of tubule segments based on the stage of spermatogenesis. This pattern of light absorption can be used to isolate specific cell associations during sperm cell differentiation.

3| Determine the light absorption pattern and identify the weak spot (stage XII-I), the strong spot (stage II-VI), the dark zone (stage VII-VIII) and the pale zone (stage IX-XI) (Fig. 1, Table 1 and Supplementary Figure 1 online).

\section{$\triangle$ CRITICAL STEPS}

For stage-specific pools of seminiferous tubules for RNA or protein analysis, see Box $\mathbf{1}$

4| Cut $<0.5-\mathrm{mm}$ piece of the stage of interest (Supplementary Video 1 online). Collect the piece

\section{BOX 1 PREPARATION OF STAGE-SPECIFIC POOLS OF THE SEMINIFEROUS TUBULES FOR BIOCHEMICAL ANALYSIS}

Cut sequential sections of $2 \mathrm{~mm}$ each beginning at the weak spot. When a sufficient amount of tubules has been dissected, transfer the pools to cryovials and remove all of the excess PBS. Freeze the pooled tubules in liquid nitrogen and transfer to $-80^{\circ} \mathrm{C}$ for long-term storage.

In our experience, $5 \mathrm{~cm}$ in total of each of the pooled stages will yield approximately $5 \mathrm{mg}$ of protein. The Petri dish should be taped to the dissecting microscope to prevent its movement and the mixing of the staged pools. To improve the accuracy of cutting the desired 2-mm length of segment, tape a ruler to the Petri dish to use as a guide for cutting the 2-mm segments of tubule. It is also useful to keep track of the number of segments cut for each grouped stage to equalize the extractions for protein and RNA analyses. To confirm that the light absorption pattern has been correctly identified, segments of tubule preceding and following the desired stage should be examined using the squash method ( $\mathbf{s e p 4 ) . ~ T o ~ f a c i l i t a t e ~ t h e ~ c o l l e c t i o n ~ o f ~ t h e ~ p o o l e d ~}$ tubules into the cryovials it is helpful to widen the mouth of the pipette tip by cutting about $1 \mathrm{~cm}$ from the end. Pipette PBS up and down a couple of times to wet the inside of the tip before collecting the tubule. To prevent RNA or protein degradation, the pooled tubules should be collected within no more than $2 \mathrm{~h}$ after sacrificing the mouse. 
of tubule in a $15-\mu$ l volume using a pipette. Transfer the tubule onto a glass slide. Carefully put a coverslip over the tubule. Avoid introducing air bubbles. The coverslip will squash the tubule and the cells will flow out (Supplementary Video 2 online). This procedure may need to be modified to improve the access of the antibodies to the antigen, in particular when studying nuclear proteins ( see Box 2).

To study molecular and cellular events during a specific stage of germ cell differentiation, a more accurate staging method using a phase-contrast microscope is required (Fig. 2).

5| Touch one edge of the coverslip with filter paper to remove extra fluid and to spread the cells (Supplementary Video 2 online).

\section{$\triangle$ CRITICAL STEPS}

6| Examine the cells with a phase

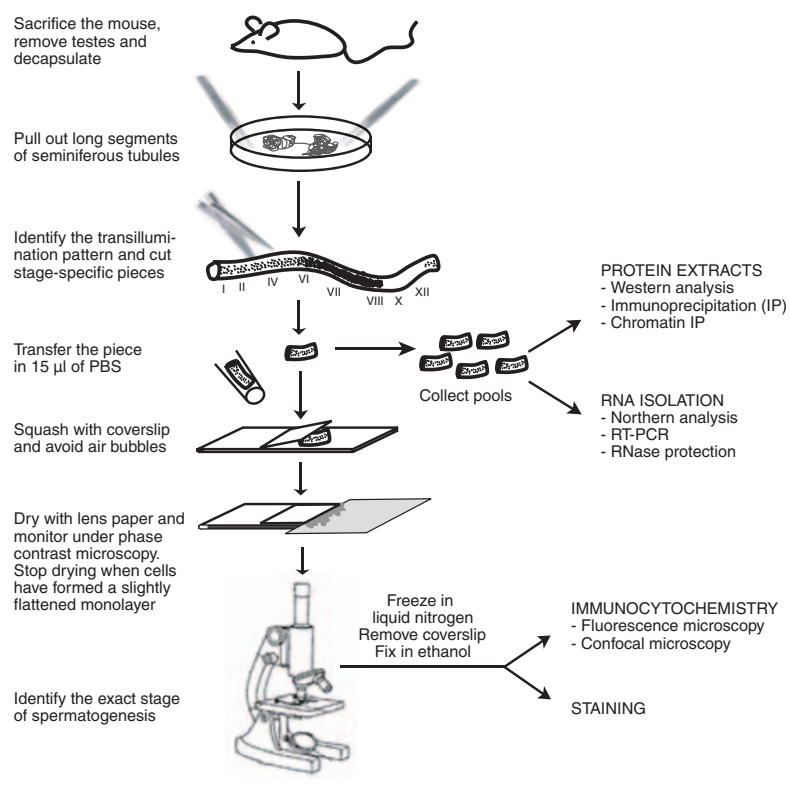

Identification of the exact stage of spermatogenesissquash preparations
Figure 2 | Illustration of the experimental procedure for the isolation of mouse spermatogenic stages and the multiple applications of this method. contrast microscope using the $40 x$

objective. Identify the stage on the basis of the cell associations and morphological characteristics.

For a more careful examination, use the $100 \times$ objective with immersion oil.

$\triangle$ CRITICAL STEPS

\section{$\Rightarrow$ TROUBLESHOOTING}

7| After examination with a microscope, freeze the slide in liquid nitrogen for $20 \mathrm{~s}$, and remove the coverslip by flipping it off with a scalpel. Fix the cells in $90 \%$ ethanol for 2-5 $\mathrm{min}$ and then air dry

(Supplementary Video 3 online). Store the slides at $15-25^{\circ} \mathrm{C}$ if they will be used within a few weeks or place at $-80^{\circ} \mathrm{C}$ for long-term storage.

\section{$\Rightarrow$ TROUBLESHOOTING}

\section{BOX 2 MODIFICATION OF SQUASH PREPARATIONS}

To improve the access of antibodies to their target antigens step 4 can be modified as follows: Cut $<0.5$ $\mathrm{mm}$ piece of the stage of interest and transfer it to a new petri dish. Add $15 \mu \mathrm{l}$ of $100 \mathrm{mM}$ sucrose to the tubule. Release the cells from the tubule either by tweezing apart the tubule or by pushing the cells out from the tubule as if emptying a sausage. Pipette up and down to resuspend the cells in the sucrose solution. Transfer the cells to a slide predipped in $1 \%$ paraformaldehyde and $0.15 \%$ Triton-X. Dry the slides in a humid chamber and then proceed directly to immunocytochemistry or freeze the slides for later use. If chromosome spreading is desired, preincubate the tubule in a hypotonic solution for $30 \mathrm{~min}$ before isolating the desired segment ${ }^{13}$. 

TABLE 1 KEY MORPHOLOGICAL FEATURES OF THE STAGE-SPECIFIC
SPERMATOGENIC CELLS

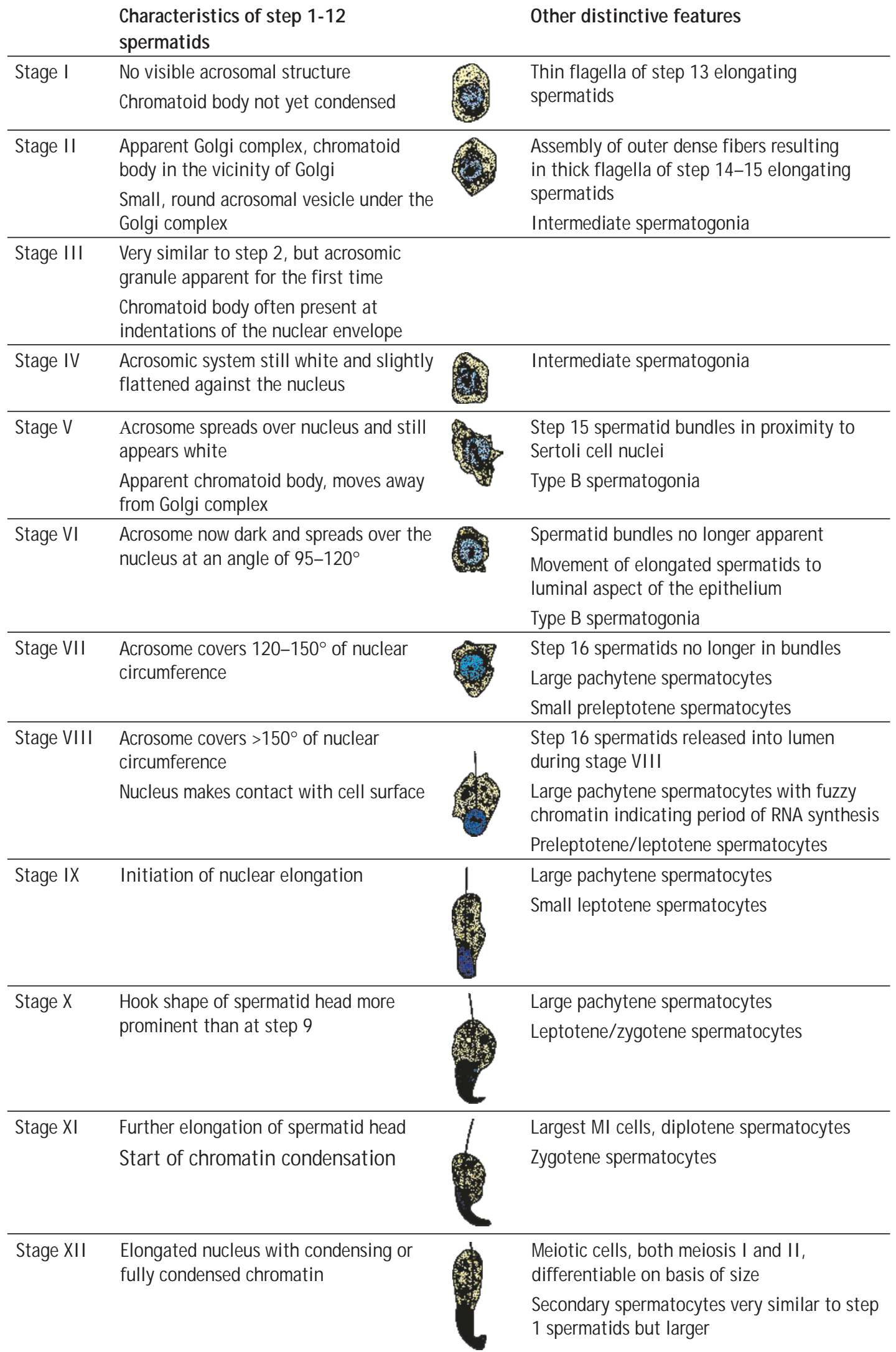




\section{TROUBLESHOOTING TABLE}

\section{PROBLEM SOLUTION}

Step 2 The tubule and the light absorption patterns are damaged during separation and cutting.

Bend one tip of the forceps to create a small hook. Lift the tubule using the hook and cut with dissection scissors. In this way you will avoid squeezing the tubule with the forceps.

Step 6 Cells in the squash preparation look unhealthy. After sacrificing the mouse, the examination of the squash preparations should be done within a maximum time of $2 \mathrm{~h}$. The appearance of pale spots in the nucleus is a typical feature of degenerating cells.

Step 6 The cells keep moving under the microscope.

Using a syringe filled with immersion oil, seal the preparation by adding a drop of immersion oil along every edge of the coverslip (Supplementary Video 2 online). This will stop the cells from moving and will prevent the sample from drying, allowing longer observation of the preparation.

Step 6 There appear to be cells representative of more than one stage.

Not all tubules will have a uniform length of waves; some will have short waves, resulting in the $0.5-\mathrm{mm}$ segment showing more than one stage.

Step 7 There is a weak signal or too much background in immunocytochemistry.

\section{Do not let slides defrost between freezing and} fixation. Squash preparations can be stored at $-80^{\circ} \mathrm{C}$, but for some antibodies, storing at $15-25^{\circ} \mathrm{C}$ (for up to 1 week) is better. Usually, the thinner the squash preparation is, the better the signal. Thus, try to cut as short a segment as possible and ensure that cells are well spread.

\section{CRITI CAL STEPS}

Step 1 It is necessary to use sexually mature mice (older than $60 \mathrm{~d}$ ) in order to see the transillumination pattern of the tubule. In juvenile mice, there is no clear transillumination pattern before the chromatin of spermatids starts to condense. But the wave organization of seminiferous epithelium is present very early in development, and it is possible to prepare squash preparations and study the morphology of the existing spermatogenic cell types in juvenile mice. All of the mouse strains that we studied have the same transillumination pattern and predicted cell associations at each stage.

Step 3 From stages XII-VI, spermatids (steps 12-16) are arranged in bundles, resulting in the speckled appearance of the tubule. Stage XII is distinguished as the weak spot, a characteristic resulting from the increased chromatin condensation of step 12 spermatids and their organization into bundles, which are observed as individual spots. Spermatid bundles are best visible in the tubular region, termed the strong spot, comprised of stages II-VI. In the periphery of the tubule, the bundles have a striped configuration because they are seen sideways, whereas in the middle of the tubules the bundles have a spotty configuration because they are seen along the axis of the bundle. This pattern reflects the deep penetration of the Sertoli cells by the spermatid bundles. As spermatogenesis progresses, the bundling of the elongating spermatids stops, coincident with the movement of step 15 spermatids toward the tubule lumen. This produces the characteristically homogenous dark zone corresponding to stages VIIVIII. Stage VIII marks the point of spermiation, and corresponds to the release of elongated spermatids from the Sertoli cells into the lumen, where they begin their voyage to the epididymis. After the point of spermiation, the dark zone abruptly changes into the pale zone, representing stages IX-X| .

Step 5 Aided by capillary diffusion that occurs when a piece of filter paper is placed at the edge of the coverslip, cells flow out of the tubule, yielding a live cell monolayer. Observe the spreading of the cells under a phase-contrast microscope and remove the filter paper when a monolayer has formed. Cells will have a slightly flattened appearance. I nsufficient drying results in a squash preparation that is too thick, making it difficult to recognize the cell types. If the squash preparation has dried too much, cells look unhealthy and damaged. 
Step 6 The stages of spermatogenesis are determined on the basis of specific cell associations (Fig. $\mathbf{1})$ and key morphological criteria (Table $\mathbf{1}$ and Supplementary Figures $\mathbf{2}$ and $\mathbf{3}$ online). The early spermatids have the most easily recognizable morphological features. The developmental state of the acrosome, nuclear shape and chromatin condensation of step 1-12 spermatids are the most commonly used hallmarks of specific stages. Additionally, the formation of flagella, the appearance of particular types of meiotic spermatocytes or spermatogonia and the possible organization of the spermatids into bundles, can be used as criteria.

\section{COMMENTS}

This method has been popularly used for the visualization of staged living spermatogenic cells in reproductive toxicology studies ${ }^{14}$, and for studying chromosome events and movements of organelles, such as the chromatoid body ${ }^{15}$ (Supplementary Video 4 online). In addition, we have pioneered new biochemical applications extending its use to studies of transcriptional regulation during spermatogenesis ${ }^{16,17}$ and to the characterization of spermatogenic gene-targeting mouse models ${ }^{18-20}$ (Supplementary Figure 4 online). The greatest challenge of this technique is the initial difficulty in learning to recognize the transillumination pattern of the seminiferous tubules and the spermatogenic cell types under phase-contrast microscopy. Our goal here was to provide clear directions and accurate descriptions of the key features used to distinguish each stage. The basics of the methodology described here have been successfully used for over 30 years by groups studying spermatogenesis around the world. Here, we have described its application for cellular and biochemical studies, and we believe that its extension to other aspects of spermatogenesis will contribute to the study of stem cell and cancer biology.

Note: Supplementary information is available on the Nature Methods website.

\section{ACKNOWLEDGMENTS}

We are grateful to R. Catena and all members of the Sassone-Corsi lab for stimulating discussions and for their contributions to this work. S. Kimmins was supported by a fellowship from the Fondation pour la Recherche Médicale, N. Kotaja by the European Molecular Biology Organization, and by Helsingin Sanomain 100-vuotis juhlasäätiö (Finland). Our studies are supported by grants from Centre National de la Recherche Scientifique, Institut National de la Santé et de la Recherche Médicale, Centre Hospitalier Universitaire Régional, Fondation de la Recherche Médicale, Université Louis Pasteur, Human Frontier Science Program (RG-240) et La Ligue contre le Cancer.

1. Sassone-Corsi, P. Unique chromatin remodeling and transcriptional regulation in spermatogenesis. Science 296, 2176-2178 (2002)

2. Russell, L.D., Ettlin, R.A., SinhaHikim, A.P. \& Clegg, E.D. Mammalian Spermatogenesis in Histological and Histopathological Evaluation of the Testis pp. 1- 40 (Cache River Press, St. Louis, 1990).

3. Perey, B., Clermont, Y. \& Leblond, C.P. The wave of the seminiferous epithelium in the rat. Am. J. Anat. 108, 47-77 (1961).

4. Parvinen, M. \& Vanha-Perttula, T. I dentification and enzyme quantitation of the stages of the seminiferous epithelial wave in the rat. Anat. Rec. 174, 435- 450 (1972).

5. Toppari, J., Eerola, E. \& Parvinen, M. Flow cytometric DNA analysis of defined stages of rat seminiferous epithelial cycle during in vitro differentiation. J. Androl. 6, 325-333 (1985).

6. Toppari, J. \& Parvinen, M. In vitro differentiation of rat seminiferous tubular segments from defined stages of the epithelial cycle morphologic and immunolocalization analysis. J. Androl. 6, 334-343 (1985).

7. Leblond, C.P. \& Clermont, Y. Definition of the stages of the cycle of the seminiferous epithelium in the rat. Ann. NY Acad. Sci. 55, 548-573 (1952).

8. Parvinen, M. Regulation of the seminiferous epithelium. Endocr. Rev. 3, 404-417 (1982).

9. Toppari, J., Bishop, P.C., Parker, J.W. \& diZerega, G.S. DNA flow cytometric analysis of mouse seminiferous epithelium. Cytometry 9, 456-462 (1988).

10. Kangasniemi, M. et al. Modulation of basal and FSH dependent cyclic AMP production in rat seminiferous tubules staged by an improved transillumination technique. Anat. Rec. 227, 62-76 (1990).

11. Parvinen, M., Toppari, J. \& Lähdetie J. Transilluminationphase contrast microscopic techniques for evaluation of male germ cell toxicity and mutagenicity. in Methods in
Reproductive Toxicology (R.E. Chapin \& J. Heindel, eds.) 142-165 (Academic Press, Orlando, Florida, USA, 1993).

12. Parvinen, M. \& Hecht, N.B. Identification of living spermatogenic cells of the mouse by transillumination-phase contrast microscopic technique for 'in situ' analyses of DNA polymerase activities. Histochemistry 71, 567-579 (1981).

13. Peters, A.H., Plug, A.W., van Vugt, M.J . \& de Boer, P. A drying-down technique for the spreading of mammalian meiocytes from the male and female germline. Chromosome Res. 5, 66-68 (1997).

14. Henriksen, K. \& Parvinen, M. Stage-specific apoptosis of male germ cells in the rat: mechanisms of cell death studied by supravital squash preparations. Tissue Cell 30, 692-701 (1998).

15. Ventelä, S., Toppari, J . \& Parvinen, M. Intercellular organelle traffic through cytoplasmic bridges in early spermatids of the rat: mechanisms of haploid gene product sharing. Mol. Biol. Cell 14, 2768-2780 (2003).

16. Macho, B. et al. CREM-dependent transcription in male germ cells controlled by a kinesin. Science 298, 2388-2390 (2002).

17. De Cesare, D., Fimia, G. M., Brancorsini, S., Parvinen, M. \& Sassone-Corsi, P. Transcriptional control in male germ cells: general factor TFIIA participates in CREM-dependent gene activation. Mol. Endocrinol. 17, 2554-2565 (2003)

18. Meng, $X$. et al. Regulation of cell fate decision of undifferentiated spermatogonia by GDNF. Science 287 . 1489-1493 (2000).

19. Martianov, I. et al. Late arrest of spermiogenesis and germ cell apoptosis in mice lacking the TBP-like TLF/ TRF2 gene. Mol. Cell 7, 509-515 (2001).

20. Martianov, l. et al. Distinct functions of TBP and TLF/ TRF2 during spermatogenesis: requirement of TLF for heterochromatic chromocenter formation in haploid round spermatids. Development 129, 945-955 (2002). 